\title{
A substrate ambiguous enzyme facilitates genome reduction in an intracellular symbiont
}

\author{
Daniel RG Price and Alex CC Wilson ${ }^{*}$
}

\begin{abstract}
Background: Genome evolution in intracellular microbial symbionts is characterized by gene loss, generating some of the smallest and most gene-poor genomes known. As a result of gene loss these genomes commonly contain metabolic pathways that are fragmented relative to their free-living relatives. The evolutionary retention of fragmented metabolic pathways in the gene-poor genomes of endosymbionts suggests that they are functional. However, it is not always clear how they maintain functionality. To date, the fragmented metabolic pathways of endosymbionts have been shown to maintain functionality through complementation by host genes, complementation by genes of another endosymbiont and complementation by genes in host genomes that have been horizontally acquired from a microbial source that is not the endosymbiont. Here, we demonstrate a fourth mechanism.

Results: We investigate the evolutionary retention of a fragmented pathway for the essential nutrient pantothenate (vitamin B5) in the pea aphid, Acyrthosiphon pisum endosymbiosis with Buchnera aphidicola. Using quantitative analysis of gene expression we present evidence for complementation of the Buchnera pantothenate biosynthesis pathway by host genes. Further, using complementation assays in an Escherichia coli mutant we demonstrate functional replacement of a pantothenate biosynthesis enzyme, 2-dehydropantoate 2-reductase (E.C. 1.1.1.169), by an endosymbiont gene, ilvC, encoding a substrate ambiguous enzyme.

Conclusions: Earlier studies have speculated that missing enzyme steps in fragmented endosymbiont metabolic pathways are completed by adaptable endosymbiont enzymes from other pathways. Here, we experimentally demonstrate completion of a fragmented endosymbiont vitamin biosynthesis pathway by recruitment of a substrate ambiguous enzyme from another pathway. In addition, this work extends host/symbiont metabolic collaboration in the aphid/Buchnera symbiosis from amino acid metabolism to include vitamin biosynthesis.
\end{abstract}

Keywords: Bacteriocyte, Symbiosis, Endosymbiosis, Co-evolution, Gammaproteobacteria

\section{Background}

Extensive genome reduction in obligate endosymbiotic bacteria is a hallmark of intracellularity [1-3]. Recent genome sequencing of diverse bacterial endosymbionts of insects reveals ongoing symbiont genome reduction, resulting in small gene dense genomes that contain a subset of genes compared to their closest free-living relatives [4-7]. For example, the genome of the gammaproteobacterium Buchnera from the aphid, Cinara cedri, is only $416 \mathrm{kbp}$, approximately 11 -fold smaller that its closest free-living relative Escherichia coli $[4,8]$. Currently, the smallest known bacterial genomes, containing fewer than 200 protein-coding genes, belong to

\footnotetext{
* Correspondence: acwilson@bio.miami.edu

Department of Biology, University of Miami, Coral Gables, FL 33146, USA
}

insect associated obligate endosymbionts $[6,7,9,10]$. Eliminated genes include those that are either functionally redundant, or not essential for maintenance of the symbiosis [1-3]. Many of the genes evolutionarily retained in endosymbiont genomes are those required for biosynthesis of nutrients essential to the host. However, even endosymbiont essential nutrient biosynthesis pathways experience gene loss such that it is common to find fragmented, but evolutionary retained, pathways that appear incomplete and non-functional [11,12].

Recent genome sequencing of holosymbionts (hosts + their symbionts) reveals that fragmented biosynthesis pathways can be completed by three processes: (1) complementation by host genes with overlapping function [13-15]; (2) complementation by genes encoded by another 
symbiont [4-6]; and (3) complementation by genes horizontally transferred to the host nuclear genome from other bacterial genomes [11,15]. All three of these processes can contribute to functional maintenance of metabolic pathways in a single endosymbiont and yet, even accounting for these three independent processes, some evolutionarily retained symbiont pathways remain fragmented making it unclear if they are functional $[11,12]$.

A fourth process for completion of fragmented biosynthesis pathways was proposed by Shigenobu et al. [12] when they hypothesized that missing enzyme steps in fragmented Buchnera amino acid biosynthesis pathways are mediated by other (unidentified) Buchnera enzymes with overlapping activity [12,14]. In addition to welldefined primary roles within linear metabolic pathways, it is common for enzymes to catalyze side reactions [16-18]. Such multifunctional enzymes that are capable of catalyzing weak side reactions, exhibit either catalytic promiscuity, catalysis of a different reaction type on a single or range of substrates; or substrate ambiguity, catalysis of the same reaction type on a range of substrates [16-18]. Extraordinary levels of metabolic plasticity within organisms are facilitated by weak side reactions [19-24]. Importantly, weak side activities provide precursor catalytic activity for the evolution of new enzyme function [16-18,21,22]. It is not without precedent that multifunctional enzymes with overlapping activity 'fill holes' in fragmented essential metabolic pathways [25-27]. For example, the fragmented folate biosynthesis pathway from the obligate intracellular pathogen Chlamydia is functional because of the recruitment of promiscuous and adaptable enzymes from other biosynthetic pathways [25].

Here, working with the best-characterized holosymbiosis, that of the pea aphid Acyrthosiphon pisum with the gammaproteobacterium Buchnera, we reveal that the fragmented Buchnera pantothenate (vitamin B5) biosynthesis pathway can be completed using a Buchnera substrate ambiguous enzyme recruited from another pathway. We demonstrate using complementation assays in E. coli mutants that retention of a substrate ambiguous enzyme in the massively reduced genome of Buchnera facilitates the collaborative biosynthesis of pantothenate (vitamin B5) in the A. pisum/Buchnera holosymbiont.

\section{Results and discussion}

Buchnera has a fragmented but evolutionary retained pantothenate biosynthesis pathway

Compared to E. coli, the obligate endosymbiont Buchnera from the pea aphid, A. pisum, has a fragmented pantothenate biosynthesis pathway that is missing panD, panE and $i l v E$ (Figure 1). Gene identifiers and enzyme activities are provided in Table 1. Buchnera with fully sequenced genomes fall into two groups: (1) those from species such as Baizongia pistaciae, C. cedri and Uroleucon ambrosiae that have lost the whole pantothenate biosynthesis pathway [4,28,29]; and (2) those from species such as A. pisum, Myzus persicae and Schizaphis graminum that retain identically fragmented pathways characterized by loss of panD, panE and ilvE [12,30,31] (Figure 1). Evolutionary retention by multiple Buchnera lineages of identically fragmented pantothenate biosynthesis pathways that include two genes (panB and panC) that only function in pantothenate biosynthesis, led us to hypothesize that the pantothenate biosynthesis pathway in these species is functional.

To begin the investigation of our hypothesis that evolutionary retention of identically fragmented pantothenate biosynthesis pathways indicates retention of pantothenate biosynthesis capacity we characterized expression of $i l v H, i l v C, i l v D, \operatorname{pan} B$ and panC in the specialized aphid bacteriocyte cells that house Buchnera. All five of these Buchnera genes were highly expressed in A. pisum bacteriocytes and consistently highly expressed across three genetically independent $A$. pisum lines (Figure 2A). Functionality of these fragmented pathways requires (1) host supply of $\beta$-alanine and (2) complementation of panE encoded 2-dehydropantoate 2-reductase (E.C. 1.1.1.169).

\section{A. pisum synthesizes $\beta$-alanine via the uracil degradation pathway in bacteriocytes}

A. pisum and other insects synthesize $\beta$-alanine via two main pathways: (1) the uracil degradation pathway [32] and (2) via decarboxylation of L-aspartate [33] (Figure 1). Gene identifiers and enzyme activities are provided in Table 1 . While synthesis of $\beta$-alanine is evolutionarily conserved in the major insect orders (Kyoto Encyclopedia of Genes and Genomes (KEGG) pathway map 00410), we propose that $\beta$-alanine biosynthesis in A. pisum is now additionally required to supply Buchnera.

Gene expression analysis demonstrates that $\beta$-alanine biosynthesis via the A. pisum uracil degradation pathway is upregulated in bacteriocytes relative to whole insect expression (Figure 2B). Consistent with uracil degradation being the major $\beta$-alanine biosynthesis route in bacteriocytes, the alternate pathway that synthesizes $\beta$-alanine via decarboxylation of L-aspartate is down regulated in bacteriocytes (Figure 2B). Aspartate decarboxylase (ADC) is 4.3-fold down in LSR1 bacteriocytes, 25 -fold down in 5A bacteriocytes and 100-fold down in 9-2-1 bacteriocytes relative to whole insect expression.

\section{Buchnera ilvC complements panE to complete the pantothenate biosynthesis pathway in $A$. pisum}

Given host supply of $\beta$-alanine, functional pantothenate biosynthesis in Buchnera with the fragmented pantothenate biosynthesis pathway requires complementation 
Buchnera APS and E. coli vitamin B5 biosynthesis

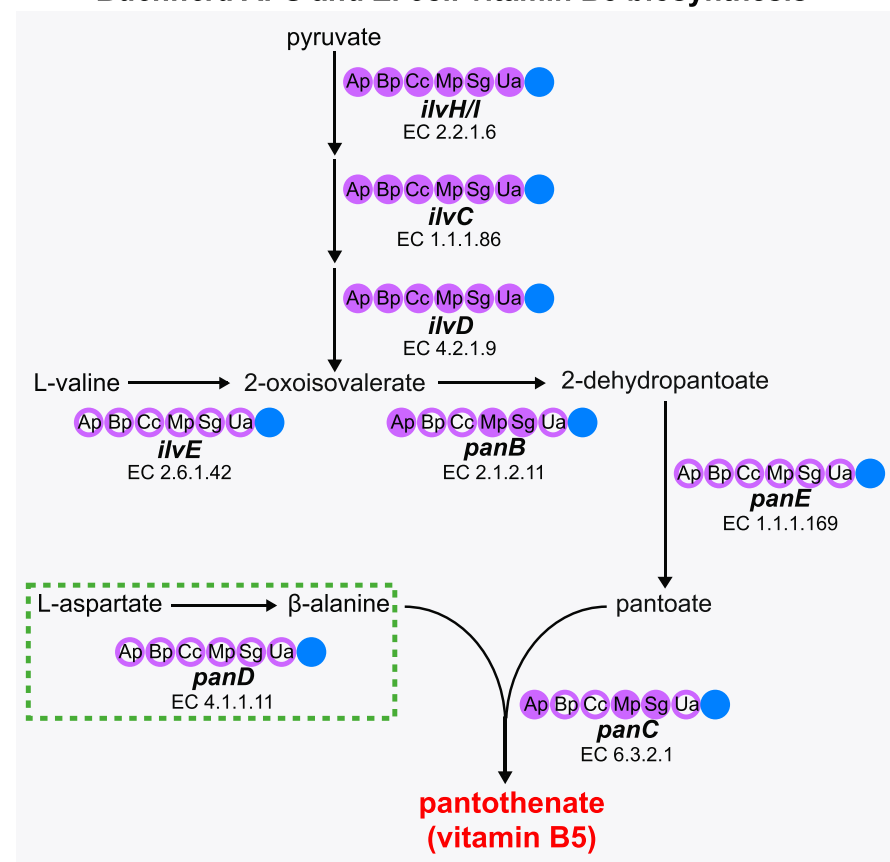

A. pisum $\beta$-alanine biosynthesis
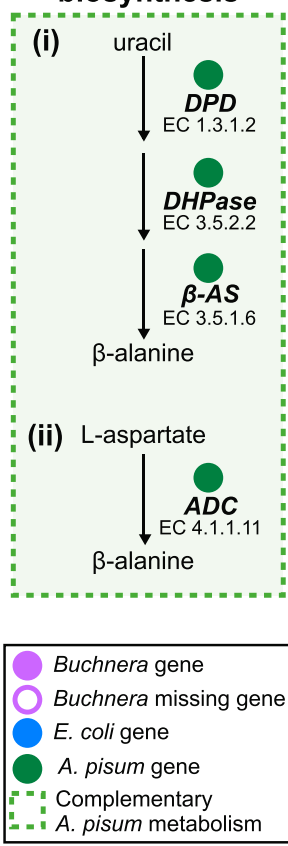

Figure 1 Comparative analysis of the Buchnera pantothenate biosynthesis pathway and complementary A. pisum $\beta$-alanine biosynthesis pathways. Metabolic pathways were reconstructed using KEGG pantothenate biosynthesis pathway from E. coli K-12 (eco00770) and from Buchnera from host aphids that included: A. pisum (Ap, buc00770), B. pistaciae (Bp, bab00770), C. cedri (Cc, bcc00770), M. persicae (Mp, [30]), S. graminum (Sg, bas00770) and U. ambrosiae (Ua, bua00770) [34,35]. Complementary $\beta$-alanine biosynthesis pathways of A. pisum (api00410) [34,35] are shown. The presence/absence of biosynthesis genes in the Buchnera, E. coli and A. pisum genomes is indicated. Full details of A. pisum, Buchnera and E. coli gene identifiers and enzyme activities are provided in Table 1. KEGG, Kyoto Encyclopedia of Genes and Genomes.

\section{(A) Buchnera pantothenate biosynthesis} $\mathrm{Ba}$

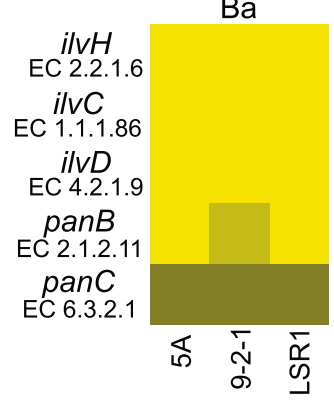

(B)

EC 1.3 .1 .2 EC 1.3.1.2 EC 3.5.2.2 BUP EC 3.5.1.6 $A D C$ EC 4.1.1.11 1

A. pisum $\beta$-alanine biosynthesis WI

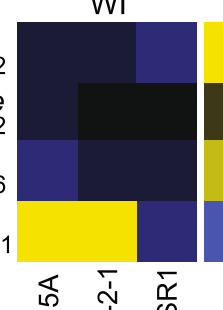

$\mathrm{Ba}$

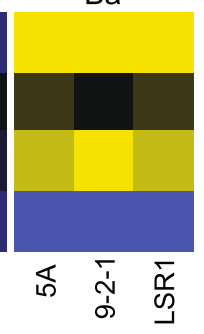

High

expression

expression

Figure 2 Quantitative expression analyses of Buchnera and A. pisum pantothenoate biosynthesis genes. Expression profiles for (A) Buchnera pantothenate biosynthesis genes and (B) complementary A. pisum $\beta$-alanine biosynthesis genes were generated for three independent A. pisum lineages (5A, 9-2-1 and LSR1). Expression of Buchnera genes in bacteriocytes (Ba) was normalized to atpE (BU003) while expression of A. pisum genes in whole insects (WI), bacteriocyte (Ba) and gut (G) tissues was normalized to GAPDH (ACYPI009769). Relative abundance of each gene was compiled into a heat map showing high expression (yellow) to low expression (blue). The box shows gene expression fold-change in A. pisum LSR1 bacteriocyte (Ba) tissues relative to whole insect (WI) expression. Fold-change was calculated according to [36]; for each aphid lineage $n=3$. 
Table 1 Pantothenate biosynthesis genes and enzyme activities

\begin{tabular}{|c|c|c|c|c|}
\hline \multicolumn{5}{|c|}{ Buchnera and E. coli vitamin B5 biosynthesis } \\
\hline Gene & Buchnera ID \# & E. coli ID \# & E.C \# & Enzyme activity (abbreviated activity) \\
\hline$i l v E$ & $x$ & EG10497 & 2.6.1.42 & branched-chain amino-acid-transaminase (BCAT) \\
\hline$i \mid V l$ & BU226 & EG10500 & 2.2.1.6 & acetolactate synthase (ALS) \\
\hline$i / V H$ & BU225 & EG10499 & 2.2.1.6 & acetolactate synthase (ALS) \\
\hline$i / V N$ & $x$ & EG10502 & 2.2.1.6 & acetolactate synthase (ALS) \\
\hline$i l v B$ & $x$ & EG10494 & 2.2.1.6 & acetolactate synthase (ALS) \\
\hline$i l v M$ & $x$ & EG10501 & 2.2.1.6 & acetolactate synthase (ALS) \\
\hline$i l v C$ & BU599 & EG10495 & 1.1.1.86 & ketol-acid reductoisomerase (KARI) \\
\hline$i l v D$ & BU600 & EG10496 & 4.2.1.9 & dihydroxyacid dehydratase (DHAD) \\
\hline panB & BU197 & EG11675 & 2.1.2.11 & ketopantoate hydroxymethltransferase (KPHMT) \\
\hline panE & $x$ & EG13271 & 1.1.1.169 & ketopantoate reductase (KPR) \\
\hline pand & $x$ & EG11747 & 4.1.1.11 & L-aspartate-a-decarboxylase (ADC) \\
\hline panc & BU196 & EG11746 & 6.3.2.1 & pantothenate synthetase (PS) \\
\hline \multicolumn{5}{|c|}{ A. pisum $\beta$-alanine biosynthesis } \\
\hline Gene & ACYPI ID \# & LOC ID \# & E.C \# & Enzyme activity (abbreviated activity) \\
\hline$D P D$ & ACYPI004747 & LOC100163680 & 1.3.1.2 & dihydropyrimidine dehydrogenase (DPD) \\
\hline DHPase $e^{a}$ & ACYPI002925 & LOC100161725 & 3.5.2.2 & dihydropyrimidinase (DHPase) \\
\hline$B \cup P$ & ACYPI003488 & LOC100162330 & 3.5.1.6 & beta-ureidopropionase (BUP) \\
\hline$A D C$ & ACYPI009960 & LOC100169332 & 4.1.1.11 & L-aspartate-a-decarboxylase (ADC) \\
\hline
\end{tabular}

${ }^{a}$ Alternative splice forms are annotated in National Center for Biotechnology Information (NCBI) A. pisum RefSeq (Acyr_2.0) gene models. Gene identification numbers (ID \#) are from: Buchnera APS [12], E. coli K-12 MG1655 (ID\# from EcoGene [37]) and A. pisum LSR1 [38]. Enzyme Commission number (E.C \#) and enzyme activity are from the BRENDA enzyme information system [39].

of lost panE encoded 2-dehydropantoate 2-reductase (E.C. 1.1.1.169). Previous work in Salmonella typhimurium [40] and E. coli [41] has shown that $i l v C$, a gene encoding ketol-acid reductoisomerase (E.C. 1.1.1.86) that mediates reactions in the biosynthesis of the essential amino acids isoleucine and valine and production of 2-oxoisovalerate from pyruvate in pantothenate biosynthesis, also has limited 2-dehydropantoate 2-reductase (E.C. 1.1.1.169) activity. While ilvC can reduce 2 -dehydropantoate to pantoate, the activity of ilvC in S. typhimurium to function in pantothenate biosynthesis is 20 -fold lower than its activity as ketol-acid reductoisomerase [40] and so low in E. coli that the ilvC knockout mutant was indistinguishable from wild-type $\mathrm{K} 12$ in its reduction of 2-dehydropantoate to pantoate [41]. However, given that generation of $E$. coli pantoate auxotrophs requires generation of $p a n E$, ilvC double mutants, we hypothesized that Buchnera ilvC has sufficient 2-dehydropantoate 2 -reductase activity and, thereby, critically maintains functional pantothenate biosynthesis in Buchnera that retain fragmented pantothenate biosysnthesis pathways.

To test the hypothesis that Buchnera ilvC has 2dehydropantoate 2-reductase activity sufficient to complete pantoate synthesis we utilized a 2-dehydropantoate 2reductase negative strain of E. coli (strain FE8: $i l v C^{-}$and panE $E^{-}$[41]) and functionally complemented E. coli FE8 with Buchnera ilvC (Figure 3A). When cells were plated on selective plates (minimal media plates, supplemented with L-isoleucine and 3-methyl-2-oxobutanoic acid to complement deletion of $i l v C$ but without pantothenate supplementation), rescue of $E$. coli FE8 was only achieved by complementation with E. coli panE and Buchnera ilvC (Figure $3 \mathrm{~A})$. In contrast, under selective conditions, very

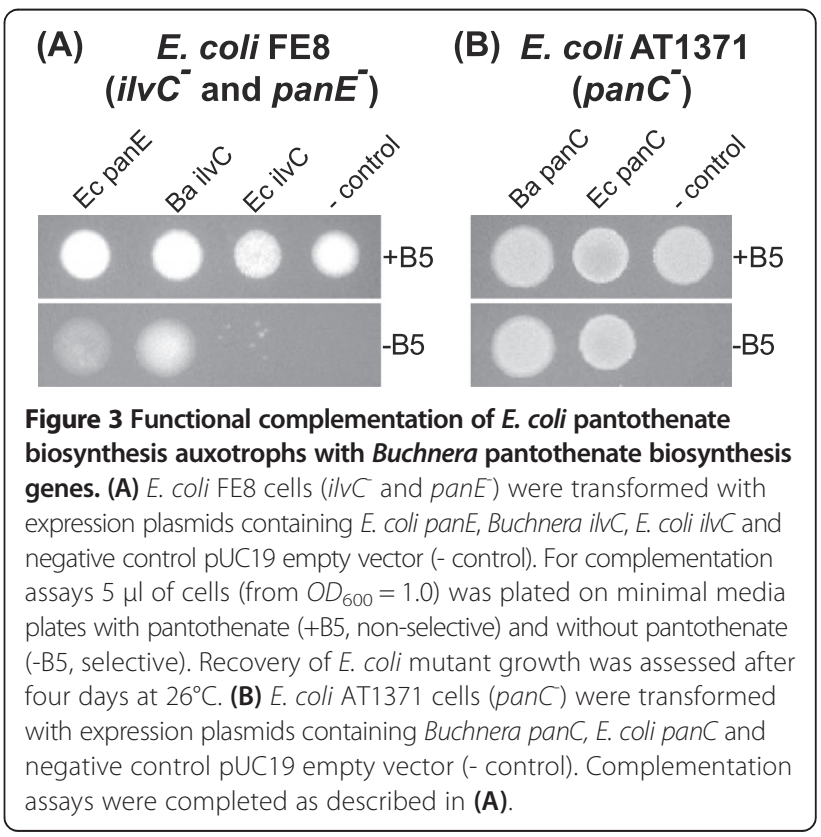


limited growth of FE8 cells transformed with E. coli ilvC was observed, suggesting that $E$. coli ilvC does not contain sufficient 2-dehydropantoate 2-reductase activity to rescue 2-dehydropantoate 2-reductase negative $E$. coli cells, a result consistent with the earlier work of Elischewski et al., [41]. As a negative control, E. coli FE8 was transformed with pUC19 empty expression vector. As expected, these pUC19 empty vector transformed cells did not grow without pantothenate supplementation (Figure 3A). When grown in non-selective conditions (minimal media plates with L-isoleucine, 3-methyl-2-oxobutanoic acid and pantothenate supplementation) FE8 cells transformed with E. coli panE, Buchnera ilvC, E. coli ilvC and pUC19 empty expression vector (negative control) established robust colonies (Figure 3A).

Functional complementation assays were also completed to test the pantoate- $\beta$-alanine ligase (E.C. 6.3.2.1) activity of Buchnera panC. Buchnera panC shares only $48 \%$ amino acid sequence identity with $E$. coli panC. Despite this high level of sequence divergence, Buchnera pan $C$ retains pantoate- $\beta$-alanine ligase activity (Figure $3 \mathrm{~B}$ ). Both Buchnera APS panC and E. coli panC (positive control) functionally rescue E. coli AT1371 (panC strain) when grown on a minimal media without pantothenate supplementation. Negative control E. coli AT1371 transformed with pUC19 empty expression vector was unable to grow on minimal media without pantothenate supplementation. With pantothenate supplementation, growth of AT1371 transformed with Buchnera panC, E. coli panC and negative control cells is observed (Figure 3B).

Loss of Buchnera ilvE facilitates holosymbiont integration Loss of Buchnera ilvE (encoding branched-chain-aminoacid transaminase, E.C. 2.6.1.42) is not critical to the integrity of the pantothenate biosynthesis pathway in Buchnera because Buchnera with fragmented pathways retain $i l v H / I$, ilvC and $i l v D$ and are predicted to synthesize 2-oxoisovalerate from pyruvate (Figure 1). However, the loss of Buchnera ilvE has important coevolutionary implications for the biosynthesis of the branched chain amino acids (isoleucine, leucine and valine). Symbiont loss of $i l v E$ was first reported in Buchnera APS from $A$. pisum [12] a decade before the genome of $A$. pisum was published [38]. While Shigenobu et al., [12] had speculated that Buchnera APS likely utilized the activity of another transaminase to complete branched chain amino acid biosynthesis, members of the Pea Aphid Genome Consortium offered an alternate explanation [42], that Buchnera loss of $i l v E$ encoded branched-chain-aminoacid transaminase is complemented by the host genome encoded branched-chain-amino-acid transaminase (BCAT, ACYPI008371, [42]). Recently, the collaborative role of BCAT in the biosynthesis of leucine, one of the three branched chain amino acids, has been confirmed experimentally [43]. Identical patterns of holosymbiont coevolution involving symbiont loss of $i l v E$ have likely also coevolved in the citrus mealybug, Planococcus citri [11], and the whitefly, Bemisia tabaci [31].

\section{Host/symbiont metabolic collaboration and a substrate ambiguous enzyme facilitate maintenance of functional pantothenate biosynthesis pathways in some Buchnera lineages}

On the basis of holosymbiont metabolic pathway analysis, gene expression analysis and complementation tests in E. coli pantothenate biosynthesis mutants, we propose a model of collaborative pantothenate biosynthesis that critically depends on host supply of $\beta$-alanine, and the substrate ambiguous activity of ketol-acid reductoisomerase (E.C. 1.1.1.86) encoded by $i l v C$ as 2-dehydropantoate 2-reductase (E.C. 1.1.1.169) (Figure 4).

A. pisum synthesize $\beta$-alanine in bacteriocytes via the uracil degradation pathway (Figure 4). To complete pantothenate biosynthesis, $\beta$-alanine is transported from the bacteriocyte cytoplasm to Buchnera via a currently unidentified transporter (Figure 4). In Buchnera's closest free-living relative $E$. coli, $\beta$-alanine is actively transported via amino acid transporter $c y c A$ [44]. However, due to extensive gene loss, Buchnera has lost almost all substrate specific transporters, including $c y c A[12,45]$. Our current working hypothesis, as previously proposed by Shigenobu et al., [12], is that Buchnera utilizes a flagellum-derived general substrate transport system for import of essential nutrients, including $\beta$-alanine.

Notably, we demonstrate that panE encoding 2dehydropantoate 2-reductase has been lost from Buchnera's genome and functionally replaced with $i l v C$, an enzyme that encodes both ketol-acid reductoisomerase (E.C. 1.1.1.86) and 2-dehydropantoate 2-reductase (E.C. 1.1.1.169) activity. The gene product of $i l v C$ functions as a substrate ambiguous reductase in branched chain amino acid biosynthesis and pantothenate biosynthesis. Functional replacement of panE encoded ketopantoate reductase with a substrate ambiguous $i l v C$ encoded ketopantoate reductase is not without precedent since loss of panE and functional replacement by $i l v C$ has previously been demonstrated in the gram-positive soil bacterium Corynebacterium glutamicum [46]. In the context of holosymbiont evolution, we hypothesize that retention of multifunctional endosymbiont enzymes facilitates genome reduction while maintaining the functional integrity of essential biosynthetic pathways, a process that facilitates genome reduction but results in increased functional complexity of symbiont-retained genes. Consistent with our hypothesis, in genome-wide analyses, it has recently been demonstrated that bacterial species with small, reduced genomes have increased protein functional complexity, leading the authors to suggest that an increase in 


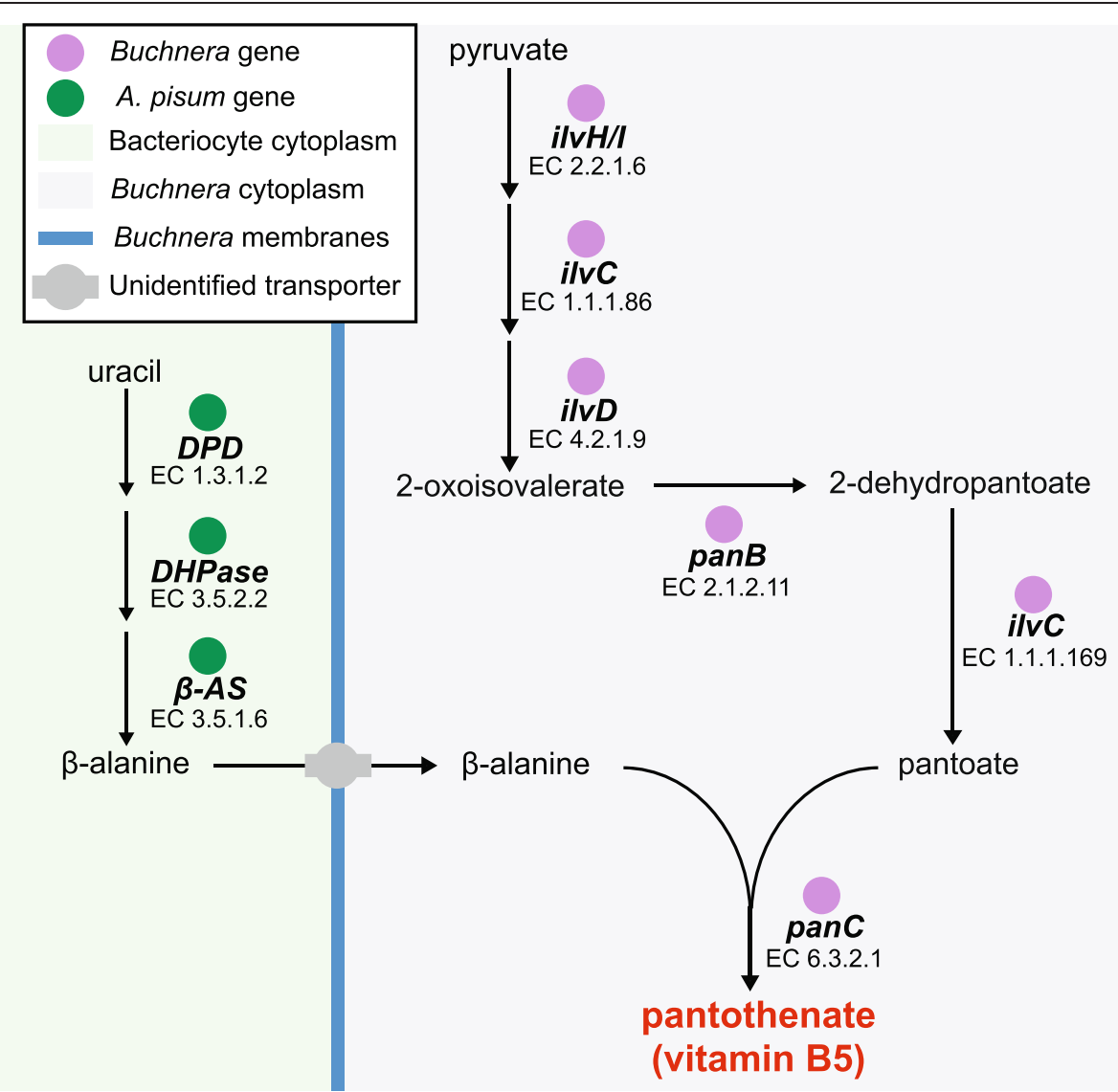

Figure 4 Pantothenate (vitamin B5) biosynthesis in the A. pisum/Buchnera holosymbiont.

functional complexity of retained genes in small genomes compensates for gene losses [47].

\section{Conclusions}

There are currently four mechanisms that facilitate retention of metabolic functionality concomitant with gene loss in the primary endosymbionts of insects. All four mechanisms can operate within a single genome and all four contribute to explaining why fragmented and disconnected biosynthesis pathways, that on first observation appear incomplete and non-functional, are retained across diverse obligate endosymbiont taxa. As more holosymbiont genomes become available, metabolic reconstruction of obligate intracellular symbionts must consider a complex evolutionary patchwork that comprises host metabolic complementarity, symbiont replacement, horizontal transfer of complementary biosynthesis genes to the host genome and now also protein multifunctionality in endosymbionts, all of which contribute to the maintenance of endosymbiont metabolic function.

\section{Methods}

Aphid culture

Parthenogenetic females of the pea aphid A. pisum, lineage LSR1 [38], 5A and 9-2-1 [48] were maintained on pre- flowering Vicia faba cv. The Sutton. All aphids were kept at $20^{\circ} \mathrm{C}$ in a long day regime of 16 hours of light and 8 hours of dark.

\section{Comparative analysis of vitamin B5 biosynthesis}

Metabolic pathways for pantothenate (vitamin B5) biosynthesis were analyzed using the KEGG pathway database $[34,35]$. These included the pantothenate biosynthesis pathways from E. coli K-12 MG1655 (eco00770) and B. aphidicola from host aphids, including: $A$. pisum (Buchnera Ap, buc00770 [12]), B. pistaciae (Buchnera Bp, bab00770 [28]), C. cedri (Buchnera Cc, bcc00770 [4]), M. persicae (Buchnera Mp [30]), S. graminum (Buchnera Sg, bas00770 [31]) and $U$. ambrosiae (Ua, bua00770 [29]). The complementary $A$. pisum $\beta$-alanine metabolism pathway was identified in A. pisum LSR1 (api00410 [38]).

\section{Real-time quantitative RT-PCR}

Real-time quantitative PCR (QPCR) was used to compare A. pisum and Buchnera pantothenate biosynthesis gene expression in whole adult female aphids and isolated bacteriocytes using $2^{-\Delta \Delta C T}$ methodology [36]. Methods are identical to those described in detail by [49]. Primers were designed using Primer3Plus software for real-time 
PCR [50], and specificity checked using Primer-BLAST against NCBI $A$. pisum RefSeq gene models (primer sequences are shown in Additional file 1: Table S1). For comparative gene expression analysis, all QPCR primers had amplification efficiencies $>90 \%$ and $<105 \%$. A. pisum gene expression was normalized to glyceraldehyde-3dehydrogenase (GAPDH, ACYPI009769) and Buchnera gene expression was normalized to atpE ATP synthase FOF1 subunit $C$ (atpE, BU003). The normalized expression value for each gene was compiled into a heat map ( $z$-score $\mathrm{x} 10 ; z=$ (each value - average)/standard deviation). Yellow: $z>0$, blue: $z<0$, black: $z=0$.

\section{ilvC, panC and panE expression constructs}

Full-length Buchnera ilvC (BU599) and panC (BU196) coding sequences were amplified from Buchnera LSR1 gDNA, and full-length E. coli ilvC (EG10495), panC (EG11746) and panE (EG13271) coding sequences were amplified from E. coli K-12 MG1655 gDNA; all coding sequences were amplified using Phusion high-fidelity DNA polymerase (Thermo Scientific, Waltham, MA, USA). Primers were designed to incorporate restriction enzyme sites to allow directional cloning of $i l v C$, pan $C$ and panE coding sequences into pUC19, in frame and downstream of the lacZ start codon; thus, generating pUC19 expression constructs containing ilvC, panC and panE coding sequences under the control of the lac $Z$ promoter (primer sequences are shown in Additional file 1: Table S1). Amplification reactions were completed on a Mastercycler PCR system (Eppendorf, Hauppauge, NY, USA) with the following conditions: $98^{\circ} \mathrm{C}$ for 30 seconds, followed by 30 cycles at $98^{\circ} \mathrm{C}$ for 10 seconds, $55^{\circ} \mathrm{C}$ for 30 seconds and $72^{\circ} \mathrm{C}$ for 1 minute. Amplified coding sequences were digested with either HindIII and BamHI, or BamHI and EcoRI (see Additional file 1: Table S1) and cloned into the respective sites of pUC19. Expression constructs containing Buchnera and E. coli ilvC coding sequences were named pBUC-ilvC and pECO-ilvC, respectively. Expression constructs containing Buchnera and $E$. coli panC coding sequences were named pBUCpanC and $\mathrm{pECO}-$ pan $C$, respectively. The expression construct containing E. coli panE coding sequence was named pECO-panE. All expression constructs were fully sequenced using Sanger sequencing in standard Big Dye terminator v3.1 reactions (Life Technologies, Green Island, NY, USA). Reaction products were analyzed on 3130xl genetic analyzer (Life Technologies), and sequence data assembled into contiguous sequences using Sequencher v4.9 (Gene Codes Corp., Ann Arbor, MI, USA).

\section{Functional complementation of E. coli auxotrophs}

Functional complement experiments were carried out using the E. coli ilvC/panE double mutant FE8 (ilvC::aacC1, panE::tetA; [41]), obtained as a kind gift from Jörn
Kalinowski (Institute for Genome Research and Systems Biology, Bielefield University, Germany); and E. coli panC mutant AT1371 ( $\mathrm{F}^{-}$, panC4, $\Delta$ (gpt-proA)62, lacY1, tsx-29, $\operatorname{gln} V 44(\mathrm{AS})$, galK2(Oc), $\lambda^{-}$, Rac-0, hisG4(Oc), rfbC1, xylA5, $m t l-1, \operatorname{argE3}(\mathrm{Oc})$, thiE1 [51,52]), obtained from the Yale University E.coli Genetic Stock Center (CGSC, Yale University, New Haven, CT, USA.).

E. coli FE8 (ilvC and panE ${ }^{-}$) cells were transformed with plasmids pBUC-ilvC, pECO-ilvC, pECO-panE and pUC19 empty vector (negative control) according to [53]. Transformants were grown overnight in Luria-Bertani (LB) media with $50 \mu \mathrm{g} / \mathrm{ml}$ ampicillin, $15 \mu \mathrm{g} / \mathrm{ml}$ gentamicin and $10 \mu \mathrm{g} / \mathrm{ml}$ tetracycline and washed twice in sterile distilled water and finally resuspended to an attenuance of $O D_{600}=1.0$. Functional complementation of $E$. coli FE8 pantothenate auxotrophs was carried out by plating aliquots $(5 \mu \mathrm{l})$ of cell suspensions on M63 minimal media agar plates [54] containing $13.6 \mathrm{~g} / \mathrm{L} \mathrm{KH}_{2} \mathrm{KO}_{4}$ (pH 7.0), $2 \mathrm{~g} / \mathrm{L}\left(\mathrm{NH}_{4}\right)_{2} \mathrm{SO}_{4}, 0.4 \%$ (w/v) glycerol, $0.25 \mathrm{~g} / \mathrm{L}$ $\mathrm{MgSO}_{4} .7 \mathrm{H}_{2} \mathrm{O}, 50 \mathrm{mg} / \mathrm{ml}$ L-isoleucine, $50 \mathrm{mg} / \mathrm{ml} \mathrm{3-}$ methyl-2-oxobutanoic acid (Acros Organics, Waltham, $\mathrm{MA}$, USA), $0.5 \mathrm{mg} / \mathrm{L} \mathrm{FeSO}_{4} .7 \mathrm{H}_{2} \mathrm{O}, 0.5 \mathrm{mg} / \mathrm{L}$ thiamine, $1.5 \%(\mathrm{w} / \mathrm{v})$ agar, $50 \mu \mathrm{g} / \mathrm{ml}$ ampicillin, $15 \mu \mathrm{g} / \mathrm{ml}$ gentamicin and $10 \mu \mathrm{g} / \mathrm{ml}$ tetracycline. Aliquots of cell suspensions were also plated on M63 minimal media agar plates supplemented with $50 \mu \mathrm{g} / \mathrm{ml} \mathrm{D-pantothenic} \mathrm{acid} \mathrm{(non-}$ selective) to assess cell viability. E. coli FE8 cell growth on selective plates (without vitamin B5) and nonselective plates (with vitamin B5) was assessed after four days of growth at $26^{\circ} \mathrm{C}$.

E. coli AT1371 (panC) cells were transformed with plasmids pBUC-panC, pECO-panC (positive control) and pUC19 empty vector (negative control) according to [53]. Transformants were grown overnight in LB media with $50 \mu \mathrm{g} / \mathrm{ml}$ ampicillin and washed twice in sterile distilled water and finally resuspended to an attenuance of OD600 = 1.0. Functional complementation of AT1371 pantothenate auxotrophs was carried out by plating aliquots $(5 \mu \mathrm{l})$ of cell suspensions on M63 minimal media agar plates [54] con-

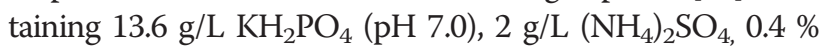
(w/v) glycerol, $0.25 \mathrm{~g} / \mathrm{L} \quad \mathrm{MgSO}_{4} .7 \mathrm{H}_{2} \mathrm{O}, \quad 0.5 \mathrm{mg} / \mathrm{L}$ $\mathrm{FeSO}_{4} .7 \mathrm{H}_{2} \mathrm{O}, 0.5 \mathrm{mg} / \mathrm{L}$ thiamine, $1.5 \%(\mathrm{w} / \mathrm{v})$ agar and $100 \mu \mathrm{g} / \mathrm{ml}$ ampicillin. Aliquots of cell suspensions were also plated on M63 minimal media agar plates supplemented with $50 \mu \mathrm{g} / \mathrm{ml} \mathrm{D}$-pantothenic acid (non-selective) to assess cell viability. E. coli FE8 cell growth on selective plates (without pantothenate) and non-selective plates (with pantothenate) was assessed after four days of growth at $26^{\circ} \mathrm{C}$.

\section{Additional file}

Additional file 1: Table S1. Pantothenate biosynthesis primers. Notes: "Alternative splice forms are annotated in NCBI A. pisum RefSeq 
(Acyr_2.0) gene models. QPCR primers were designed to amplify all splice forms. Restriction enzyme (RE) sites for forward (fwd) and reverse (rev) expression construct primers are shown in bold.

\section{Competing interests}

Both authors declare that they have no competing interests.

\section{Authors' contributions}

DRGP and ACCW conceived of and designed the study and wrote the paper. DRGP performed all experimental work. Both authors read and approved the final manuscript.

\section{Authors' information}

DRGP is a molecular biologist who uses functional genomic approaches to characterize the interaction between phloem-feeding insects and their obligate endosymbiotic bacteria. ACCW studies genome coevolution between plant sap-feeding insects and their obligate intracellular bacterial symbionts. Recent work in Wilson's group focuses on functional characterization of amino acid transporters in sternorrhynchan insects.

\section{Acknowledgements}

We are grateful to Philip Kushlan for useful discussion. This work was supported by National Science Foundation Award 1121847 (to ACCW and DRGP).

Received: 18 September 2014 Accepted: 11 December 2014 Published online: 20 December 2014

\section{References}

1. McCutcheon JP: The bacterial essence of tiny symbiont genomes. Curr Opin Microbiol 2010, 13:73-78.

2. Moran NA, Bennett GM: The tiniest tiny genomes. Annu Rev Microbiol 2014, 68:195-215

3. McCutcheon JP, Moran NA: Extreme genome reduction in symbiotic bacteria. Nat Rev Microbiol 2012, 10:13-26.

4. Pérez-Brocal V, Gil R, Ramos S, Lamelas A, Postigo M, Michelena JM, Silva FJ, Moya A, Latorre A: A small microbial genome: the end of a long symbiotic relationship? Science 2006, 314:312-313.

5. Lamelas A, Gosalbes MJ, Manzano-Marín A, Peretó J, Moya A, Latorre A: Serratia symbiotica from the aphid Cinara cedri: a missing link from facultative to obligate insect endosymbiont. PLoS Genet 2011, 7:1-11.

6. McCutcheon JP, von Dohlen CD: An interdependent metabolic patchwork in the nested symbiosis of mealybugs. Curr Biol 2011, 21:1366-1372.

7. Bennett GM, Moran NA: Small, smaller, smallest: the origins and evolution of ancient dual symbioses in a phloem-feeding insect. Genome Biol Evol 2013, 5:1675-1688

8. Gil R, Sabater-Munoz B, Latorre A, Silva FJ, Moya A: Extreme genome reduction in Buchnera spp.: toward the minimal genome needed for symbiotic life. Proc Natl Acad Sci U S A 2002, 99:4454-4458.

9. McCutcheon JP, McDonald BR, Moran NA: Origin of an alternative genetic code in the extremely small and GC-rich genome of a bacterial symbiont. PLoS Genet 2009, 5:e1000565.

10. Nakabachi A, Yamashita A, Toh H, Ishikawa H, Dunbar HE, Moran NA, Hattori M: The 160-kilobase genome of the bacterial endosymbiont Carsonella. Science 2006, 314:267.

11. Husnik F, Nikoh N, Koga R, Ross L, Duncan RP, Fujie M, Tanaka M, Satoh N, Bachtrog D, Wilson ACC, von Dohlen CD, Fukatsu T, McCutcheon JP: Horizontal gene transfer from diverse bacteria to an insect genome enables a tripartite nested mealybug symbiosis. Cell 2013, 153:1567-1578.

12. Shigenobu S, Watanabe H, Hattori M, Sakaki Y, Ishikawa H: Genome sequence of the endocellular bacterial symbiont of aphids Buchnera sp. APS. Nature 2000, 407:81-86.

13. Hansen AK, Moran NA: Aphid genome expression reveals host-symbiont cooperation in the production of amino acids. Proc Natl Acad Sci U S A 2011, 108:2849-2854.

14. Shigenobu S, Wilson ACC: Genomic revelations of a mutualism: the pea aphid and its obligate bacterial symbiont. Cell Mol Life Sci 2011, 68:1297-1309.

15. Sloan DB, Nakabachi A, Richards S, Qu J, Murali SC, Gibbs RA, Moran NA: Parallel histories of horizontal gene transfer facilitated extreme reduction of endosymbiont genomes in sap-feeding insects. Mol Biol Evol 2014, 31:857-871.

16. Jensen RA: Enzyme recruitment in evolution of new function. Annu Rev Microbiol 1976, 30:409-425.

17. Khersonsky O, Tawfik DS: Enzyme promiscuity: a mechanistic and evolutionary perspective. Annu Rev Biochem 2010, 79:471-505.

18. O'Brien PJ, Herschlag D: Catalytic promiscuity and the evolution of new enzymatic activities. Chem Biol 1999, 6:R91-R105.

19. D'Ari R, Casadesus J: Underground metabolism. BioEssays 1998, 20:181-186.

20. Carbonell P, Lecointre G, Faulon JL: Origins of specificity and promiscuity in metabolic networks. J Biol Chem 2011, 286:43994-44004.

21. Nam H, Lewis NE, Lerman JA, Lee DH, Chang RL, Kim D, Palsson BO: Network context and selection in the evolution to enzyme specificity. Science 2012, 337:1101-1104.

22. Notebaart RA, Szappanos B, Kintses B, Pál F, Györkei A, Bogos B, Lázár V, Spohn R, Csörgő B, Wagner A, Ruppin E, Pál C, Papp B: Network-level architecture and the evolutionary potential of underground metabolism. Proc Natl Acad Sci U S A 2014, 111:11762-11767.

23. Yip SH, Matsumura I: Substrate ambiguous enzymes within the Escherichia coli proteome offer different evolutionary solutions to the same problem. Mol Biol Evol 2013, 30:2001-2012.

24. Greenspan RJ: The flexible genome. Nat Rev Genet 2001, 2:383-387.

25. Adams NE, Thiaville JJ, Proestos J, Juárez-Vázquez AL, McCoy AJ, Barona-Gómez F, Iwata-Reuyl D, de Crécy-Lagard V, Maurelli AT: Promiscuous and adaptable enzymes fill "holes" in the tetrahydrofolate pathway in Chlamydia species. MBio 2014, 5:e01378-01314.

26. Tian J, Bryk R, Shi S, Erdjument-Bromage H, Tempst P, Nathan C: Mycobacterium tuberculosis appears to lack a-ketoglutarate dehydrogenase and encodes pyruvate dehydrogenase in widely separated genes. Mol Microbiol 2005, 57:859-868.

27. Wagner T, Bellinzoni M, Wehenkel A, O'Hare HM, Alzari PM: Functional plasticity and allosteric regulation of a-ketoglutarate decarboxylase in central mycobacterial metabolism. Chem Biol 2011, 18:1011-1020.

28. van Ham RC, Kamerbeek J, Palacios C, Rausell C, Abascal F, Bastolla U, Fernández JM, Jiménez L, Postigo M, Silva FJ, Tamames J, Viguera E, Latorre A, Valencia A, Morán F, Moya A: Reductive genome evolution in Buchnera aphidicola. Proc Natl Acad Sci U S A 2003, 100:581-586.

29. Degnan $\mathrm{PH}$, Ochman $\mathrm{H}$, Moran NA: Sequence conservation and functional constraint on intergenic spacers in reduced genomes of the obligate symbiont Buchnera. PLoS Genet 2011, 7:e1002252.

30. Jiang Z, Jones DH, Khuri S, Tsinoremas NF, Wyss T, Jander G, Wilson ACC: Comparative analysis of genome sequences from four strains of the Buchnera aphidicola Mp endosymbion of the green peach aphid. Myzus persicae. BMC Genomics 2013, 14:917.

31. Tamas I, Klasson L, Canbäck B, Näslund AK, Eriksson AS, Wernegreen JJ, Sandström JP, Moran NA, Andersson SG: 50 million years of genomic stasis in endosymbiotic bacteria. Science 2002, 296:2376-2379.

32. Piskur J, Schnackerz KD, Andersen G, Bjornberg O: Comparative genomics reveals novel biochemical pathways. Trends Genet 2007, 23:369-372.

33. Richardson G, Ding H, Rocheleau T, Mayhew G, Reddy E, Han Q, Christensen BM, Li J: An examination of aspartate decarboxylase and glutamate decarboxylase activity in mosquitoes. Mol Biol Rep 2010, 37:3199-3205.

34. Kanehisa M, Goto S: KEGG: Kyoto Encyclopedia of Genes and Genomes. Nucleic Acids Res 2000, 28:27-30.

35. Kanehisa M, Goto S, Sato Y, Furumichi M, Tanabe M: KEGG for integration and interpretation of large-scale molecular data sets. Nucleic Acids Res 2012, 40:D109-D114.

36. Livak KJ, Schmittgen TD: Analysis of relative gene expression data using real-time quantitative PCR and the $2^{-\Delta \Delta C T}$ method. Methods 2001, 25:402-408

37. Zhou J, Rudd KE: EcoGene 3.0. Nucleic Acids Res 2013, 41:D613-D624.

38. International Aphid Genomics Consortium: Genome sequence of the pea aphid Acyrthosiphon pisum. PLOS Biol 2010, 8:e1000313.

39. Schomburg I, Chang A, Placzek S, Söhngen C, Rother M, Lang M, Munaretto C, Ulas S, Stelzer M, Grote A, Scheer M, Schomburg D: BRENDA in 2013: integrated reactions, kinetic data, enzyme function data, improved disease classification: new options and contents in BRENDA. Nucleic Acids Res 2013, 41:D764-D772.

40. Primerano DA, Burns RO: Role of acetohydroxy acid isomeroreductase in biosynthesis of pantothenic acid in Salmonella typhimurium. J Bacteriol 1983, 153:259-269. 
41. Elischewski F, Puhler A, Kalinowski J: Pantothenate production in Escherichia coli K12 by enhanced expression of the panE gene encoding ketopantoate reductase. J Biotechnol 1999, 75:135-146.

42. Wilson ACC, Ashton PD, Calevro F, Charles H, Colella S, Febvay G, Jander G, Kushlan PF, Macdonald SJ, Schwartz JF, Thomas GH, Douglas AE: Genomic insight into the amino acid relations of the pea aphid, Acyrthosiphon pisum, with its symbiotic bacterium Buchnera aphidicola. Insect Mol Biol 2010, 19:249-258.

43. Russell CW, Bouvaine S, Newell PD, Douglas AE: Shared metabolic pathways in a coevolved insect-bacterial symbiosis. Appl Environ Microbiol 2013, 79:6117-6123.

44. Schneider F, Kramer R, Burkovski A: Identification and characterization of the main $\beta$-alanine uptake system in Escherichia coli. Appl Microbiol Biotechnol 2004, 65:576-582.

45. Charles H, Balmand S, Lamelas A, Cottret L, Pérez-Brocal V, Burdin B, Latorre A, Febvay G, Colella S, Calevro F, Rahbé Y: A genomic reappraisal of symbiotic function in the aphid/Buchnera symbiosis: reduced transporter sets and variable membrane organisations. PloS One 2011, 6:e29096.

46. Merkamm M, Chassagnole C, Lindley ND, Guyonvarch A: Ketopantoate reductase activity is only encoded by ilvC in Corynebacterium glutamicum. J Biotechnol 2003, 104:253-260.

47. Kelkar YD, Ochman H: Genome reduction promotes increase in protein functional complexity in bacteria. Genetics 2013, 193:303-307.

48. Dunbar HE, Wilson ACC, Ferguson NR, Moran NA: Aphid thermal tolerance is governed by a point mutation in bacterial symbionts. PLOS Bio/ 2007, 5:1006-1015.

49. Price DRG, Duncan RP, Shigenobu S, Wilson ACC: Genome expansion and differential expression of amino acid transporters at the aphid/Buchnera symbiotic interface. Mol Biol Evol 2011, 28:3113-3126.

50. Untergasser A, Nijveen $H$, Rao X, Bisseling T, Geurts R, Leunissen JAM: Primer3Plus, an enhanced web interface to Primer3. Nucleic Acids Res 2007, 35:W71-W74.

51. Cronan JE: $\beta$-alanine synthesis in Escherichia coli. J Bacteriol 1980, 141:1291-1297.

52. Bukhari Al, Taylor AL: Genetic analysis of diaminopimelic acid- and lysine-requiring mutants of Escherichia coli. J Bacteriol 1971, 105:844-854

53. Tauch A, Kirchner O, Wehmeier L, Kalinowski J, Puhler A: Corynebacterium glutamicum DNA is subjected to methylation-restriction in Escherichia coli. FEMS Microbiol Lett 1994, 123:343-347.

54. Elbing K, Brent R: Media preparation and bacteriological tools. Curr Protoc Mol Biol 2002, Chapter 1:Unit 1.1. doi:10.1002/0471142727.mb0101s59.

\section{Submit your next manuscript to BioMed Central and take full advantage of:}

- Convenient online submission

- Thorough peer review

- No space constraints or color figure charges

- Immediate publication on acceptance

- Inclusion in PubMed, CAS, Scopus and Google Scholar

- Research which is freely available for redistribution 